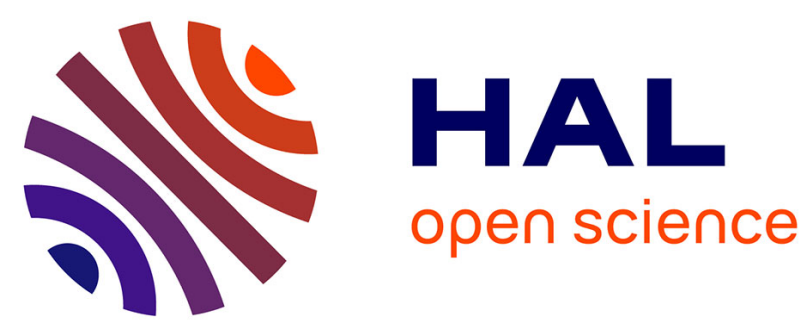

\title{
Efficient second harmonic generation in nanophotonic waveguides for optical signal processing
}

Kevin Lenglé, Laurent Bramerie, Mathilde Gay, Jean-Claude Simon, Sylvain Combrié, Gaëlle Lehoucq, Alfredo de Rossi

\section{- To cite this version:}

Kevin Lenglé, Laurent Bramerie, Mathilde Gay, Jean-Claude Simon, Sylvain Combrié, et al.. Efficient second harmonic generation in nanophotonic waveguides for optical signal processing. Applied Physics Letters, 2013, 102, pp.151114. 10.1063/1.4802790 . hal-00845310

\section{HAL Id: hal-00845310 https://hal.science/hal-00845310}

Submitted on 29 Jul 2013

HAL is a multi-disciplinary open access archive for the deposit and dissemination of scientific research documents, whether they are published or not. The documents may come from teaching and research institutions in France or abroad, or from public or private research centers.
L'archive ouverte pluridisciplinaire HAL, est destinée au dépôt et à la diffusion de documents scientifiques de niveau recherche, publiés ou non, émanant des établissements d'enseignement et de recherche français ou étrangers, des laboratoires publics ou privés. 


\title{
Efficient Second Harmonic Generation in Nanophotonic Waveguides for Optical Signal Processing
}

\author{
K. Lenglé, ${ }^{1,2}$ L. Bramerie, ${ }^{1,2}$ M. Gay, ${ }^{1,2}$ J-C. Simon, ${ }^{1,2}$ S. Combrié, ${ }^{3}$ G. Lehoucq, ${ }^{3}$ and A. De Rossi ${ }^{3, a)}$ \\ ${ }^{1)}$ Université Européenne de Bretagne (UEB), 5 Bld. Laennec, 35000 Rennes, \\ France \\ ${ }^{2)}$ CNRS-Foton Laboratory (UMR 6082), Enssat, BP 80518, 22305 Lannion, France \\ 3) THALES Research and Technology, 1 Av. A. Fresnel, 91767 Palaiseau, France
}

(Dated: 10 April 2013)

We report very efficient second harmonic generation in membrane photonic crystal waveguide. The combination of absorption-free material and slow-light enhancement enables a large harmonic generation efficiency. A peak pump power of about $450 \mathrm{~mW}$ generates up to $45 \mu W$ peak second harmonic power over a broad spectral range. The signal is exploited to demonstrate optical performance monitoring of data at $42 \mathrm{~Gb} / \mathrm{s}$.

Harmonic generation and, in particular, Second Harmonic Generation (SHG) is a very important nonlinear effect in optics. Because of their large intrinsic nonlinear susceptibility, semiconductor waveguides have been proposed for efficient frequency conversion and harmonic generation ${ }^{1}$. Furthermore, semiconductors offer a powerful technological platform, enabling the integration of photonic circuits. However, SHG is only possible in noncentrosymmetric materials, such as III-Vs or strained silicon $^{2}$. More recently, two-dimensional air-cladded Photonic Crystals $(\mathrm{PhC})$ have emerged as ideal materials for enhancing the light-matter interaction, hence the nonlinear response, owing to their remarkable light confining capability. In particular, Third Harmonic Generation (THG) has been demonstrated in Silicon $\mathrm{PhCs}^{3}$ and, later, exploited to perform optical signal monitoring ${ }^{4}$.

SHG has first been observed in $\mathrm{InP} \mathrm{PhC}$ cavities $^{5}$ and, more recently, in Silicon $\mathrm{PhC}$, where the much weaker second order susceptibility at the semiconductor surface is enhanced resonantly in suitably designed cavities ${ }^{6}$, however the SHG power is in the $p W$ range. Efficient SHG is possible in waveguides ${ }^{7}$, however this is only true if the phase matching between the fundamental and the second harmonic is ensured, which is notoriously difficult in semiconductors. Indeed, phase-matched optical parametric interactions (including SHG) have been demonstrated owing to the so-called form-birefringence in alternating layers of GaAlAs and oxidized $\mathrm{AlAs}^{1}$, or, more recently, multi-layers of III-V alloys serving as vertical Bragg mirror ${ }^{8}$ or photonic wires ${ }^{9}$. It must however be pointed out that, as in any resonant interaction, phase matching implies a much reduced bandwidth, depending on the length of the interaction. PhCs only support leaky modes at the second harmonic ( $\mathrm{SH})$ with short lifetime, compared to the fundamental mode. As a consequence, the interaction length is much shorter and this tends to reduce the efficiency, with respect to a properly phase matched second harmonic generation process entailing properly waveguided modes. Second harmonic

\footnotetext{
a)Electronic mail: alfredo.derossi@thalesgroup.com
}

generation has been demonstrated in short $(\mathrm{L}=17 \mu m)$ $\mathrm{GaP}$ waveguides ${ }^{10}$, but, despite the slow-light enhancement, the efficiency was limited to $5 \times 10^{-7} W^{-1}$.

We demonstrate that the choice of a long waveguide $(1.2 \mathrm{~mm})$ enables a larger SHG efficiency $2 \times 10^{-4} W^{-1}$, compared to (hence more than two orders of magnitude larger, roughly scaling with the device length), which we exploit to monitor the quality of high-rate data signals (up to $42.5 \mathrm{~Gb} / \mathrm{s}$ ).

The device is a $1.2 \mathrm{~mm}$-long $\mathrm{PhC}$ waveguide made of a self-standing, $180 \mathrm{~nm}$ thick membrane of Indium Gallium Phoshpide, which is transparent up to photon energy of $1.9 \mathrm{eV}(\lambda \approx 650 \mathrm{~nm})$. The waveguide consist in a missing line defect in the regular triangular lattice of holes; the size and the position of the first row of holes have been modified in order to change the dispersion, as shown in ref. ${ }^{11}$. The input and output fibre are coupled using tapers ${ }^{12}$, shown in Fig. 1a. As a result, the total insertion loss (from the input to the output fibre, hence including coupling and waveguide propagation losses) are reduced to a minimum of about $8 \mathrm{~dB}$, away from the slow light regime, with fluctuations from waveguide to waveguide of about $1 \mathrm{~dB}$. Taking into account coupling loss of about $3 \mathrm{~dB} /$ facet, we estimate the minimum propaga-

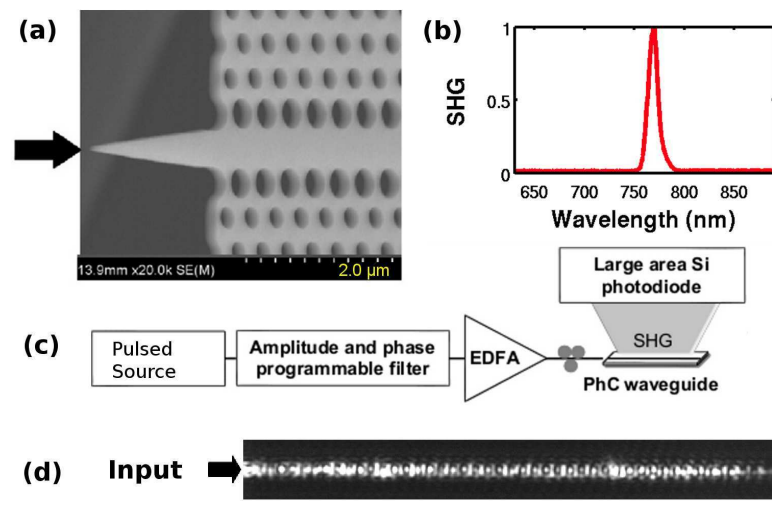

FIG. 1. (a) SEM image of the $\mathrm{PhC}$ waveguide with taper at the input; (b) detected SHG spectrum; (c) experimental setup; (d) Optical image of the entire waveguide using a SiliconCCD, revealing the SHG emission. 
tion loss to less than $2 \mathrm{~dB} / \mathrm{mm}$. Thus, in this regime, the pump intensity is weakly attenuated constant over the whole device length.

The optical input are 100 ps pulses generated from a continuous-wave tunable diode laser and a Mach-Zenhder (MZI) modulator. As the peak power is above hundreds of $\mathrm{mW}$, the radiation emitted from the top of the waveguide is easily detected by a standard silicon CCD camera, as shown in Fig. 1c,d. The peak at $770 \mathrm{~nm}$ of the emitted spectra, collected with a large aperture optical fibre $(\mathrm{NA} \approx 0.5)$, is consistent with SHG from the pump input centered at $1540 \mathrm{~nm}$ (Fig. 1b). Photonic crystal waveguides are highly dispersive and it is therefore expected that the SHG has a complex dependence on the wavelength.

In order to elucidate this point, we consider two different waveguide designs (see ref. ${ }^{11}$ ) and we report the SHG signal as a function of the wavelength. This is shown in Fig. 2a. First we note that waveguide \#1 reveals an abrupt increase of the group index from a minimum of about 6 to 24, while in waveguide \#2 the group index increases slowly from 5 to about 10. A slow-light enhancement of the SHG is expected, similarly to what has been reported for Third Harmonic Generation in Photonic Crystals ${ }^{3}$. More precisely, the theory of SHG in $\mathrm{PhC}^{13}$ predicts a quadratic dependence of the SHG efficiency $\left(\eta=P_{S H} / P_{F F}^{2}\right)$ on the group index of the fundamental mode $n_{g f}$, which can be cast as:

$$
\eta \propto d_{e f f}^{2} \omega^{2} \frac{n_{g f}^{2} n_{g s}}{A_{e f f}}
$$

Here, $d_{e f f}$ is the relevant projection of the second order susceptibility (for GaInP $110 \mathrm{pm} / \mathrm{V}$ was reported in the spectral range considered here $\left.{ }^{14}\right), A_{\text {eff }}$ is the nonlinear cross section related to the field distribution, which coincides with the geometrical cross section in the limit of plane waves. Indeed, Fig. 2c reveals a very strong dependence of the SHG signal on the wavelength, as well as a very strong correlation with the group index. Moreover, this holds for both waveguides, in spite of their different design and dispersion. A more careful inspection of the figure reveals that the peak of efficiency does not coincide with the peak of the group index. This is not surprising, because of the complex dependence of the SHG on various parameters. Moreover, it must be pointed out that a local minimum (-6dB of additional loss, Fig. 2b) of the transmission is located at the local maximum of the group index, thereby compensating for the slow-down enhancement.

This is remarkable, as, given the complex structure of the high frequency bands of the $\mathrm{PhC}$, one would expect a strong dispersive contribution both from $A_{\text {eff } f}$ and $\Delta k$. Yet, a similar correlation of the SHG with the group index has been reported in third harmonic generation in photonic crystal waveguides ${ }^{15}$. The reason why the phase mismatch term is not playing a role as crucial as it is common in nonlinear waveguides is elucidated in ref. ${ }^{15}$. Here it is shown that a (quasi) phase-matching condition is in-
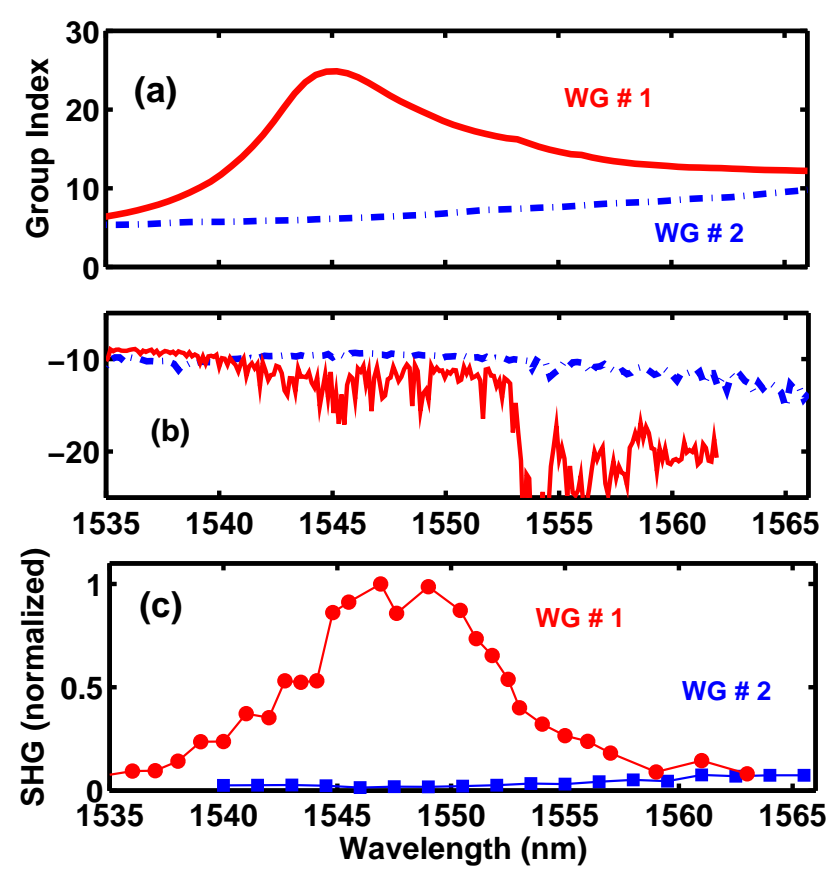

FIG. 2. (a) Dispersion (group index vs. wavelength) for two different samples (\#1,\#2); (b) Corresponding transmission spectra; (c) Corresponding detected SHG signal from the top, divided by the square of the coupled pump power.

deed governing the nonlinear interaction. Here, "quasi" is referred to the fact that the interaction length $L$ is typically much shorter than the device length, due to the short propagation length of the Bloch modes at the $\mathrm{SH}$ frequency. The short interaction length eases the phasematching condition. We believe that this also holds for the SHG process in our waveguides.

Quantitative measurements of the SHG efficiency have been performed on sample \#3 with pump wavelength set at $\lambda=1535 \mathrm{~nm}$ and $\lambda=1550 \mathrm{~nm}$ (slow light regime). A large area Si photodiode, mounted on top of the waveguide with effective collection half angle of 73 degrees, was used to measure the SHG. The coupled input peak power is increased from $10 \mathrm{~mW}$ up to about $1 \mathrm{~W}$. From $10 \mathrm{~mW}$ to 100mW a 10 Gbit/s Non Return to Zero (NRZ) Pseudo Random Binary Sequence (PRBS) is set such that the duty cycle is 0.5 . Under these conditions, the waveguide dispersion is always negligible. At larger input power, the duty cycle is decreased to $1 / 20$. The SHG peak power (Fig. 3) nicely scales with the square of the pump power over 4 orders of magnitude at least. The deviation from the ideal square law are partly due to the residual drift of the MZ modulator, leading to change in the transmitted power, but also to some thermal effects. The normalized efficiency $\eta$, referred to the peak values of the optical power (both for $\mathrm{FF}$ and $\mathrm{SH}$ ), is equal to $10^{-5} W^{-1}$ when the pump wavelength is $1535 \mathrm{~nm}$, and increases to about $2 \times 10^{-4} W^{-1}$ at a wavelength of $1550 \mathrm{~nm}$, hence a 20-fold enhancement, corresponding to roughly a 4-fold enhancement of the group index. This is consistent with 
the square scaling discussed above, also considering that losses and the nonlinear cross section $\left(A_{e f f}\right)$ also play a role here. In particular, propagation losses, which are highly dispersive in $\mathrm{PhCs}$, tend to be large at the local maximum of $n_{g}(1545 \mathrm{~nm})$, as shown in Fig. 3, inset. This explains why a larger efficiency is found at a slightly longer wavelength. The table I provides a summary of recent achievements in SHG in integrated optics. In order to allow a fair comparison, we translate the reported values into the conversion efficiency $\eta$, where the pump power refers to the peak value in pulsed experiments. As the devices have very different geometries, we report their length. The maximum SH is reported as a peak value in pulsed experiments. Where available, the SHG bandwidth, referred to the pump wavelength, is also reported. The bandwidth is defined as the tuning range of a $\mathrm{CW}$ pump (see Parameswaran et al. ${ }^{7}$ ). In the case of cavityenhanced SHG, the bandwidth is $\lambda / Q$. Finally, it must be pointed out that external conversion efficiencies have been reported here (except for Abolghasem et al. ${ }^{18}$ ). A proper correction for the extraction efficiency is too complicated to be carried out consistently.

In terms of the generated SHG power, the maximum achieved here is $45 \mu \mathrm{W}$ (peak), which is orders of magnitude larger than any previous results in nanophotonic devices. Only Bragg waveguides ${ }^{18}$ enable larger peak SHG, however, the conversion efficiency $\eta$ is very low. In contrast the normalized efficiency we report here $\eta=2 \times 10^{-4} W^{-1}$ is 2 orders of magnitude larger than what reported in III-V PhC waveguides ${ }^{10}$ and one order of magnitude larger than Si cavities, still offering a large bandwidth (Fig. 2b). In semiconductor photonics, only $\mathrm{AlGaAs}$ wires and waveguides offer better efficiency, but

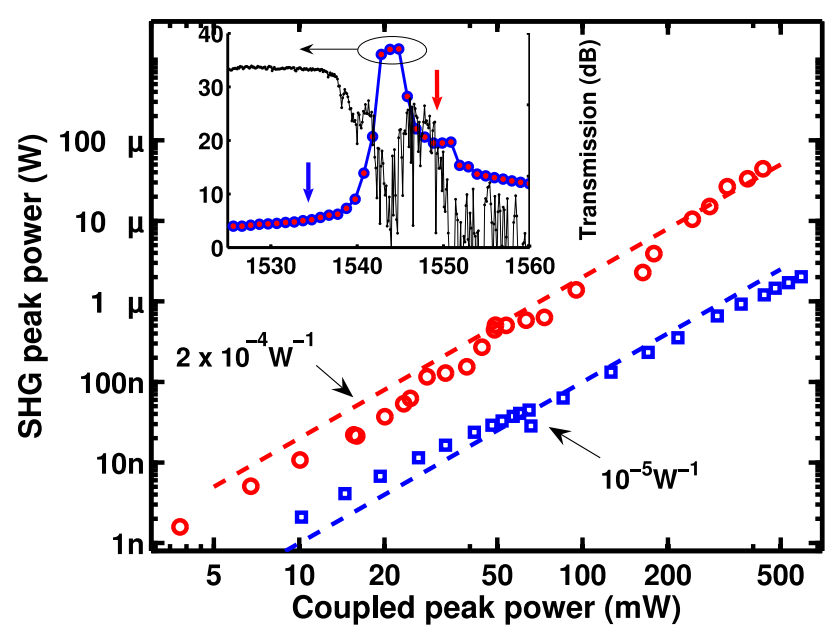

FIG. 3. Waveguide \# 3. SHG peak power vs. coupled pump peak power for $\lambda_{p}=1535 \mathrm{~nm}$ (blue, squares) and $\lambda_{p}=1550$ $\mathrm{nm}$ (red, crosses). The corresponding square laws $P_{S H}=$ $\eta\left(n_{g}\right) P_{F F}^{2}$ are shown (dashed lines). The duty cycle is $1 / 2$ at low coupled peak power (below $70 \mathrm{~mW}$ ) and $1 / 20$ at high peak power. The corresponding dispersion and transmission of the waveguide is shown in the inset.
TABLE I. Comparison of SHG in various integrated optics devices.

\begin{tabular}{lccccc}
\hline \hline Technology & $\mathrm{L}(\mathrm{mm})$ & $\eta\left(W^{-1}\right)$ & $P_{S H}$ (peak) & BW & ref. \\
\hline LiNbO $_{3}$ wg & 60 & 14 & large & $>0.25 \mathrm{~nm}$ & \\
GaP PhC wg & 0.017 & $5 \times 10^{-7}$ & $1 \mathrm{nW}$ & N.A. & 10 \\
InP PhC cav. & N.A. & N.A. & $10 \mathrm{fW}$ & N.A. & 5 \\
Si PhC cav. & N.A. & $4 \times 10^{-5}$ & $50 \mathrm{fW}$ & $50 \mathrm{pm}$ & 6 \\
GaN $\mu$-ring & N.A. & $2 \times 10^{-4}$ & $2 \mu W$ & $0.5 \mathrm{~nm}$ & 9 \\
AlGaAs wires & 1.2 & $2 \times 10^{-3}$ & $80 \mathrm{pW}$ & N.A. & 16 \\
AlGaAs wg & 1.5 & $7 \times 10^{-3}$ & $1.5 \mathrm{nW}$ & $0.3 \mathrm{~nm}$ & 17 \\
AlGaAs wg & 2.2 & $2 \times 10^{-7}$ & $190 \mu W$ & $0.8 \mathrm{~nm}$ & 18 \\
this work & 1.2 & $2 \times 10^{-4}$ & $45 \mu W$ & $\approx 10 \mathrm{~nm}$ & \\
\hline \hline
\end{tabular}

at the expense of a narrower bandwidth. Only LithiumNiobate waveguide technology enable much larger efficiency and almost $100 \%$ conversion $^{7}$, still with a relatively large bandwidth. It must be pointed out, however, that the integration of $\mathrm{LiNbO}_{3}$ waveguides with semiconductor photonics is not straightforward.

The level of the generated optical signal is more than convenient to implement optical performance monitoring of an amplitude-coded signal ${ }^{19}$. The main idea is that the second harmonic generation being dependent on the peak to average power ratio, which is easily connected to the noise level, as well as to the dispersion-induced pulse broadening. In the following, we proceed similarly as Corcoran et al., who implemented this concept by exploiting the third harmonic generation in photonic crystals ${ }^{4}$.

We consider a Return to Zero data signal at $42.5 \mathrm{~Gb} / \mathrm{s}$ with duty cycle $=33 \%$. Under these conditions, the (average) SHG signal is $0.3 \mu W, 25 \mathrm{~dB}$ above the noise floor. This holds when the pump wavelength is between $1530 \mathrm{~nm}$ and $1540 \mathrm{~nm}$. In particular, at 1533 $\mathrm{nm}$, the dispersion of the sample used here ( $\mathrm{wg} \# 4)$ is about $-0.7 \mathrm{ps}^{2} / \mathrm{mm}$ and the group index is about 17 . The coupled average power is $30 \mathrm{~mW}$. As a comparison, we also considered another wavelength $(1560 \mathrm{~nm})$ as the worst case, where the waveguide enters into propagation regime caracterized by strong attenuation and multiple scattering ${ }^{20}$.

We first characterized the impact of the dispersion. A programmable phase and amplitude optical filter is used to introduce a linear chirp (thereby simulating the propagation through a dispersive link) and an attenuator is used to decrease the Optical Signal to Noise Ratio. Figure 4a reports the dependence of the SHG on the amount of dispersion introduced, while the average input power is kept constant. As expected, this results into a pronounced decrease of the SHG signal. Interestingly, we observe a periodic behavior with the dispersion, which is due to the temporal Talbot effect ${ }^{19,21}$, thereby implying that the pulse train re-emerges again from a highly dispersed signal.

The impact of the dispersion on the SHG process has been modelled, with remarkable agreement with the ex- 

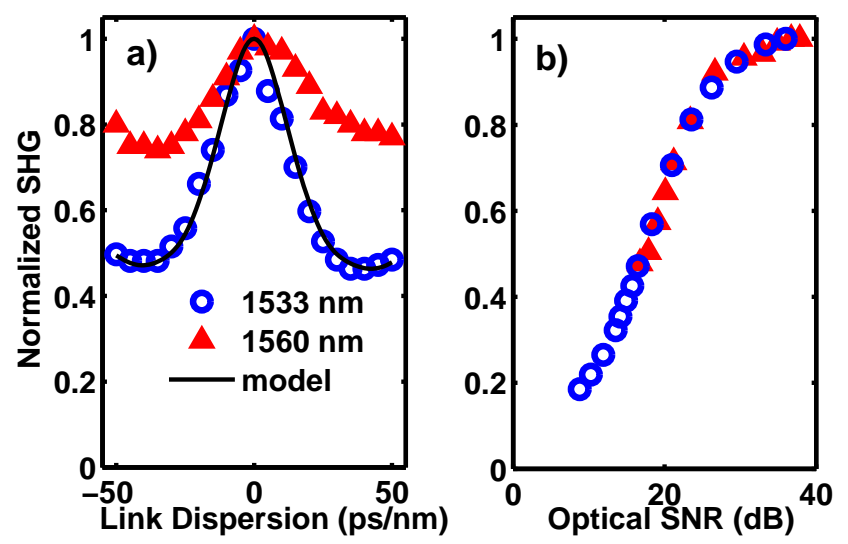

FIG. 4. Waveguide \#4. Dependence of the SHG signal on the link dispersion (a) and on the optical signal to noise ratio (b). The signal center wavelength is either $1533 \mathrm{~nm}$ (circles) or $1560 \mathrm{~nm}$ (triangles).

perimental results, in particular at $1533 \mathrm{~nm}$. The measurement at longer wavelength $(1560 \mathrm{~nm})$ deviates from the model, which is not surprising as the waveguide has very bad propagation properties here. Yet the plot keeps its bell-like shape. Fig. 4b connects the SHG signal to the optical SNR, the input average power being constant. The same monotonic increase of the SHG signal with the OSNR is observed independently on the wavelength, e.g. over the whole telecom C-band. In ref. ${ }^{4}$ similar measurements have been performed with a sensitive photodiode which detected a monitoring signal level of about 10pW, whereas our GaInP waveguide generates 300nW (average) from a roughly similar input power level. We infer therefore that the monitoring of signals with - $20 \mathrm{~dB}$ lower power level should be possible, e.g. with peak power in the $1 \mathrm{~mW}$ range.

In conclusion, we report broadband and efficient SHG (45 $\mu W$, with $450 \mathrm{~mW}$ coupled peak power and with $3 \mathrm{~dB}$ bandwidth of about $10 \mathrm{~nm}$ ) in a Photonic Crystal waveguide. The normalized conversion efficiency is $2 \times 10^{-4} W^{-1}$, which, owing to the very strong optical confinement and the slow-light enhancement, is very high for a nanophotonic device. The SHG signal has been used to monitor the OSNR and the chromatic dispersion of a high bit rate $(42.5 \mathrm{~Gb} / \mathrm{s} \mathrm{RZ})$ signal over the entire Cband.

We acknowledge the support by the European Union,
FP7/ICT funding program, under the Copernicus project (249012) (www. copernicusproject.eu). This work is also supported by Region Bretagne. We thank I. Sagnes for assistance with sample processing, P. Colman for contributions early on, S. Trillo and Y. Dumeige for enlightening discussions.

${ }^{1}$ A. Fiore, V. Berger, E. Rosencher, P. Bravetti, and J. Nagle, Nature 391, 463 (1998).

${ }^{2}$ M. Cazzanelli, F. Bianco, E. Borga, G. Pucker, M. Ghulinyan, E. Degoli, E. Luppi, V. Vniard, S. Ossicini, D. Modotto, S. Wabnitz, R. Pierobon, and L. Pavesi, Nat Mater 11, 148 (2012).

${ }^{3}$ B. Corcoran, C. Monat, C. Grillet, D. J. Moss, B. J. Eggleton, T. P. White, L. O'Faolain, and T. F. Krauss, Nat. Photon. 4, 206 (2009).

${ }^{4}$ B. Corcoran, C. Monat, M. Pelusi, C. Grillet, T. White, L. OFaolain, T. Krauss, B. Eggleton, and D. Moss, Optics express 18, 7770 (2010)

${ }^{5}$ M. W. McCutcheon, J. F. Young, G. W. Rieger, D. Dalacu, S. Frédérick, P. J. Poole, and R. L. Williams, Phys. Rev. B 76, 245104 (2007).

${ }^{6}$ M. Galli, D. Gerace, K. Welna, T. F. Krauss, L. O'Faolain, G. Guizzetti, and L. C. Andreani, Opt. Express 18, 26613 (2010).

${ }^{7}$ K. R. Parameswaran, R. K. Route, J. R. Kurz, R. V. Roussev, M. M. Fejer, and M. Fujimura, Opt. Lett. 27, 179 (2002).

${ }^{8}$ A. S. Helmy, Opt. Express 14, 1243 (2006).

${ }^{9}$ C. Xiong, W. Pernice, K. K. Ryu, C. Schuck, K. Y. Fong, T. Palacios, and H. X. Tang, Opt. Express 19, 10462 (2011).

${ }^{10}$ K. Rivoire, S. Buckley, F. Hatami, and J. Vuckovic, Applied Physics Letters 98, 263113 (2011).

${ }^{11}$ P. Colman, S. Combrié, G. Lehoucq, and A. D. Rossi, Opt. Express 20, 13108 (2012).

${ }^{12}$ Q. V. Tran, S. Combrié, P. Colman, and A. D. Rossi, Applied Physics Letters 95, 061105 (2009).

${ }^{13}$ R. Iliew, C. Etrich, T. Pertsch, and F. Lederer, Phys. Rev. B 77, 115124 (2008).

${ }^{14}$ Y. Ueno, V. Ricci, and G. I. Stegeman, J. Opt. Soc. Am. B 14, 1428 (1997).

${ }^{15}$ C. Monat, C. Grillet, B. Corcoran, D. J. Moss, B. J. Eggleton, T. P. White, and T. F. Krauss, Opt. Express 18, 6831 (2010).

${ }^{16}$ D. Duchesne, K. A. Rutkowska, M. Volatier, F. Légaré, S. Delprat, M. Chaker, D. Modotto, A. Locatelli, C. D. Angelis, M. Sorel, D. N. Christodoulides, G. Salamo, R. Arès, V. Aimez, and R. Morandotti, Opt. Express 19, 12408 (2011).

${ }^{17}$ S. Ducci, L. Lanco, V. Berger, A. D. Rossi, V. Ortiz, and M. Calligaro, Applied Physics Letters 84, 2974 (2004).

${ }^{18}$ P. Abolghasem, J. Han, B. J. Bijlani, and A. S. Helmy, Opt. Express 18, 12681 (2010).

${ }^{19} \mathrm{~S}$. Wielandy, M. Fishteyn, and B. Zhu, Lightwave Technology, Journal of 22, 784 (2004).

${ }^{20}$ M. Patterson, S. Hughes, S. Combrié, N.-V.-Q. Tran, A. D. Rossi, R. Gabet, and Y. Jaouën, Physical Review Letters 102, 253903 (2009).

${ }^{21}$ J. Azaña and M. Muriel, Opt. Lett. 24, 1672 (1999). 